\title{
INTELIGENCIA EMOCIONAL Y EMPRENDIMIENTO: POSIBLES LÍNEAS DE TRABAJO"
}

\author{
Antonia Mercedes García-Cabrera** \\ María de la Cruz Déniz-Déniz **** \\ Deybbi G. Cuéllar-Molina ${ }^{* * * * * *}$
}

* Doi: 10.11144/Javeriana.cao28-51.ieep. El artículo se recibió el 07/02/2014 y se aprobó el 15/11/2015. Sugerencia de citación: García-Cabrera, A. M.; M. C. Déniz-Déniz y D. G. Cuéllar-Molina (2015). Inteligencia emocional y emprendimiento: posibles líneas de trabajo. Cuadernos de Administración, 28 (51), 65-101. http://dx.doi.org/10.11144/Javeriana.cao28-51.ieep.

** Doctora en Ciencias Económicas y Empresariales de la Universidad de Las Palmas de Gran Canaria, España, 1994. Directora del Departamento de Economía y Dirección de Empresas de la Universidad de Las Palmas de Gran Canaria, España.

Correo electrónico: antonia.garcia@ulpgc.es

*** Doctora en Ciencias Económicas y Empresariales de la Universidad de Las Palmas de Gran Canaria, España, 1999. Profesora Titular de Universidad del Departamento de Economía y Dirección de Empresas de la Universidad de Las Palmas de Gran Canaria, España.

Correo electrónico: mariadelacruz.deniz@ulpgc.es

**** Doctora en Ciencias Económicas y Empresariales de la Universidad de Las Palmas de Gran Canaria, España, 2011. Profesora del Departamento de Economía y Dirección de Empresas de la Escuela Universitaria de Turismo de Lanzarote, adscrita a la ULPGC. Lanzarote, España.

Correo electrónico: deybbi.cuellar@ulpgc.es 


\section{Inteligencia emocional y emprendimiento: posibles líneas de trabajo}

\begin{abstract}
Resumen
Muchos estudios han intentado identificar los factores que explican la decisión emprendedora atendiendo a criterios racionales - desde la Teoría económica y la visión basada en los recursos-que ignoran el componente no racional inherente. El presente trabajo acude a la inteligencia emocional (IE) para explicar por qué algunos individuos son emprendedores mientras que otros no lo son, y encuentra que la investigación en este campo es escasa, fragmentada y, en algunos casos, contradictoria. Para avanzar en esta línea de investigación deben desarrollarse investigaciones empíricas sustentadas en diseños longitudinales y basadas en técnicas de medición de la IE trianguladas y, por tanto, más objetivas y fiables.
\end{abstract}

Palabras clave: inteligencia emocional, emprendimiento, rasgos personales. Clasificación JEL: L26, M13, M59

\section{Emotional intelligence and entrepreneurship: possible enquiry lines}

\section{Inteligência emocional e empreendimento: possiveis linhas de trabalho}

\section{ABstract}

Many research studies have attempted to identify the antecedents of entrepreneurial behavior and have tried to find them on the basis of rational criteria -Economic theory and resource-based view- that have overlooked the existence of non-rational criteria which are also behind this behavior. This work studies literature on emotional intelligence (EI) to explain why some individuals take entrepreneurial decisions and finds that research in this field is scarce, scattered, fragmented, and, in some cases, mixed or contradictory. In order to make progress in this line of research, empirical research supported on linear designs and based on more reliable triangular El measuring techniques must be carried out.

Keywords: emotional intelligence; entrepreneurship; personality traits.

JEL Classification: L26, M13, M59

\section{Resumo}

Muitos estudos vêm tentando identificar os fatores que explicam a decisão empreendedora baseando-se em critérios racionais -a teoria econômica e a visão baseada nos recursos-, que ignoram o componente não racional inerente. Este trabalho recorre à inteligência emocional (IE) como alternativa para explicar por que alguns indivíduos empreendem e outros não, e constata que a pesquisa nesse campo é escassa, dispersa, fragmentada e, em alguns casos, contraditória. Para que essa linha de pesquisa progrida, devem ser desenvolvidas pesquisas empíricas sustentadas em desenhos longitudinais e baseadas em técnicas de medição da IE trianguladas e, portanto, mais objetivas e confiáveis.

Palavras-chave: inteligência emocional, empreendimento, traços pessoais. 


\section{Introducción}

El emprendimiento ha sido objeto de gran interés en el campo de la investigación por su capacidad para dinamizar el crecimiento económico (Yang y Dess, 2007). El entorno dinámico, global y desafiante que afrontan los empresarios y directivos en la actualidad requiere que estos sean emprendedores si desean que sus empresas sobrevivan. En este contexto, los investigadores han pretendido de forma creciente comprender los antecedentes de la orientación emprendedora y sus consecuencias en términos de la contribución al crecimiento potencial de la empresa. Esto es especialmente importante en el ámbito de las pequeñas y medianas empresas (PYME), pues el emprendimiento en la misma puede proporcionarle una ventaja distintiva sobre sus rivales que sustente su supervivencia. Sin embargo, el conocimiento de los factores que hacen que los empresarios y directivos actúen de forma emprendedora es aún limitado, siendo necesario nuevas aportaciones en este ámbito (Awwad y Ali, 2012).

El emprendimiento representa un fenómeno social que ha sido abordado y tratado de explicar desde diferentes perspectivas epistemológicas (Martínez-Campos y Cantillo-Guerrero, 2013), destacando entre ellas las de naturaleza contextual y socioeconómica, las relativas a los rasgos del individuo, y más recientemente la Biología o la Neurociencia, entre otras (Nicolaou y Shane, 2014). Desde diversas teorías económicas (North, 1990; Schumpeter, 1934) se ha analizado como antecedente del emprendimiento la existencia de un entorno favorable -ej. ayudas públicas para la puesta en marcha, tasas fiscales favorables-; desde la visión basada en los recursos (Barney, 1986) el interés se ha centrado en la disponibilidad de estos por parte del emprendedor para fundar el negocio. No obstante, el proceso del emprendimiento y la forma que el emprendedor da al negocio escapa en muchos casos a los argumentos procedentes de ambas teorías de base económica (Rae, 2006). De hecho, Stevenson y Jarillo (1990) plantean que el emprendimiento es el proceso de perseguir una oportunidad sin importar los recursos controlados por el emprendedor y Zahra, Korri y Yu (2005) afirman que el emprendedor toma sus decisiones basándose tanto en criterios racionales como no racionales. Por consiguiente, y aunque los factores contextuales tengan un efecto significativo sobre la actividad emprendedo$\mathrm{ra}$, el fenómeno del emprendimiento se refiere a individuos. Por tanto, las diferencias individuales han de ser enfatizadas al identificar a los emprendedores y revelar cuáles de ellos son los más exitosos (Naktiyok, Karabey y Gulluce, 2010).

Múltiples investigadores en el campo de la creación de empresas han intentado identificar las características distintivas del individuo emprendedor frente a aquél que no lo es, al 
asumir que la persona es el elemento central en el proceso emprendedor (Pihie y Bagheri, 2010). Así, desde la Teoría de rasgos se sostiene que los atributos de personalidad son los que conducen a una persona a crear una empresa (Kuratko, 2007) -ej.: necesidad de logro (McClelland 1961), autoeficacia (Moriano, Topa, Molero, Entenza y Lévy-Mangin, 2012), locus de control, creatividad, autoestima o la capacidad de asumir riesgos (McClelland, 1961). Otros trabajos han buscado una respuesta en factores demográficos tales como la edad, el género y las experiencias previas que pueden desencadenar un comportamiento emprendedor (Louw, Van Eeden, Bosch y Venter, 2003), y algunos se han basado en características como la formación o background familiar (Shepherd, 2011). Sin embargo, ha sido común cuestionar la fiabilidad y validez de la personalidad y de las características demográficas como predictores del comportamiento emprendedor debido a los hallazgos contrapuestos y a la naturaleza estática de las características de la personalidad (Naktiyok et al., 2010).

Como extensión de la Teoría de rasgos, nosotros recurrimos a la literatura sobre Inteligencia Emocional (IE) para explicar el comportamiento emprendedor. Si bien la IE puede representar un antecedente de la personalidad del individuo, en términos conceptuales representa una variable diferente. En efecto, la llegada del concepto de IE al campo del management a principios del siglo XX (Zakarevičius y Župerka, 2010) ofrece una senda alternativa para explicar por qué algunos individuos emprenden; sin embargo, se ha prestado poca atención a esta línea de investigación (Ahmetoglu, Leutner, Chamorro-Premuzic, 2011; Neghabi, Yousefi y Rezvani, 2011).

Ante la evidencia que sugiere que la IE podría predecir los resultados en el trabajo, es razonable esperar que este constructo también prediga otras formas de éxito en la carrera profesional de un individuo (Ahmetoglu et al., 2011). Varios autores han argumentado que la IE puede constituir un predictor de los resultados del emprendimiento (Zampetakis, Beldekos y Moustakis, 2009a; Neghabi et al., 2011), por lo que puede tratarse del factor olvidado en las investigaciones sobre emprendimiento (Cross y Travaglione, 2003). Dado que la IE puede estar correlacionada con el éxito de los emprendedores, es relevante analizar esta relación. En esta línea, planteamos como objetivo de este estudio conocer la relación existente entre la IE del individuo y su comportamiento emprendedor, tratando de discernir si se trata de una relación directa o indirecta, es decir, a través de otros factores organizativos o personales que vienen influenciados por la IE. A este objetivo respondemos de forma deductiva y, tras una revisión de la literatura, formulamos proposiciones de investigación que ofrecen posibles líneas de investigación al campo. 


\section{Anotación metodológica}

La revisión bibliográfica llevada a cabo para localizar las aportaciones científicas que sustentaran el trabajo se realizó en dos fases: en la primera, nuestro propósito fue hallar los trabajos seminales que permitirían profundizar en la comprensión del emprendimiento, la inteligencia emocional y la relación entre ambos constructos, por lo que el período temporal introducido como restricción en los buscadores fue amplio y comprendió los años 1980-2012. Y en una segunda fase, se llevó a cabo una búsqueda para un período temporal más corto: 2010 a 2015, con el propósito de garantizar la inclusión de las aportaciones científicas más actuales y asociadas al estudio de los constructos y sus relaciones. En ambas fases de la búsqueda se consultaron las bases de datos disponibles en la Biblioteca digital de la Universidad de Las Palmas de Gran Canaria. En nuestras búsquedas incluimos, tanto bases de datos que contienen publicaciones en lengua castellana (Dialnet, Bases de datos del CSIC, Latindex, Scopus), como aquellas otras con publicaciones principalmente en lengua inglesa (Web of Science, Scopus, Directory of Open Access Journals, Google scholar) y bases de datos que incluían tesis doctorales (ProQuest Dissertations \& Theses Global). Entre las palabras clave que se introdujeron en las búsquedas destacan las siguientes: "emprendimiento", "emprendedor", "inteligencia emocional", "emprendimiento [e] inteligencia emocional", "emprendedor [e] inteligencia emocional", "emprendimiento [y] neurociencia" o "inteligencia emocional [y] neurociencia", siendo utilizadas éstas o sus correspondientes traducciones al inglés, según la búsqueda efectuada.

El elevado número de trabajos resultante para estas búsquedas (11.544 fueron identificados para la palabra clave emprendimiento y 15.647 para inteligencia emocional) nos llevó a refinar las mismas introduciendo nuevas restricciones. Así, se desecharon aquellos trabajos que no habían sido catalogados bajo la categoría "Management", "Business" o "Psicología", los que se correspondían con actas de congresos, por ser estos últimos en menor medida avalados por la comunidad científica, o bien aquellos que abordaban sólo tangencialmente el tema que nos ocupa. Asimismo, y a raíz de un estudio profundo de los textos seleccionados, se identificaron otros trabajos de interés que fueron debidamente localizados y analizados para la redacción del marco teórico.

Como resultado de la labor de búsqueda, se localizaron 2.408 textos de los cuales se seleccionaron para la elaboración del presente trabajo 117 de elevada relevancia científica y/o que aportaban hallazgos recientes. Estos trabajos se corresponden con los referenciados 
en la bibliografía del presente artículo. Concretamente, 88 de ellos son artículos científicos, 21 libros, 5 tesis doctorales y 3 working papers o textos publicados en Conference proceedings que por la temática específica que abordaban fueron incluidos. La calidad de estos trabajos utilizados queda de manifiesto en la Tabla 1, donde se relacionan con el número de veces en que han sido citados.

\section{Tabla 1}

Relevancia científica de los trabajos revisados

\begin{tabular}{|c|c|c|}
\hline & Autores & № citas \\
\hline \multirow{24}{*}{ 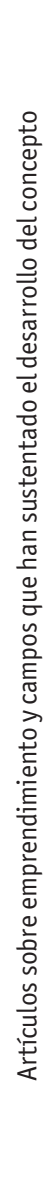 } & Ahmad, Yusoff, Noor y Ramin (2012) & 11 \\
\hline & Ahmad, Abdul Halim y Mohamed Zainal (2010) & 33 \\
\hline & Brandstätter (2011) & 112 \\
\hline & Brundin, Patzelt y Shepherd (2008) & 104 \\
\hline & Cassis y Minoglou (2005) & 59 \\
\hline & Chell y Baines (2000) & 425 \\
\hline & Chen, Greene y Crick (1998) & 1.605 \\
\hline & Cullen, Johnson y Parboteeah (2014) & 12 \\
\hline & Ensley, Pearce y Hmieleski (2006) & 185 \\
\hline & Foo (2011) & 10 \\
\hline & Guiso y Rustichini (2011) & 20 \\
\hline & Jayawarna, Rouse y Kitching (2013) & 67 \\
\hline & Johnson (2009) & 10 \\
\hline & Kalantaridis (2004) & 39 \\
\hline & Krueger (2005) & 351 \\
\hline & Kuratko (2007) & 144 \\
\hline & Lee, Wong, Foo y Leung (2011) & 171 \\
\hline & Li, Chen, Liu y Peng (2014) & 24 \\
\hline & Lim, Morse, Mitchell y Seawright (2010) & 93 \\
\hline & Lindquist, Sol y Van Praag (2012) & 26 \\
\hline & Low y MacMillan (2007) & 7 \\
\hline & Louw, Van Eeden, Bosch y Venter (2003) & 53 \\
\hline & Lumpkin y Dess (1996) & 5.800 \\
\hline & Man y Lau (2000) & 143 \\
\hline
\end{tabular}




\begin{tabular}{|c|c|c|}
\hline & Autores & № citas \\
\hline \multirow{27}{*}{ 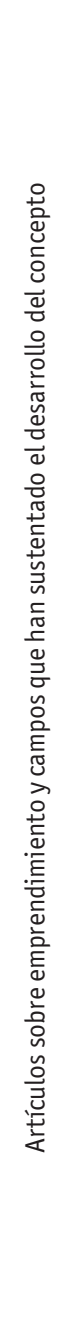 } & Martin de Holan (2014) & 4 \\
\hline & Marvel (2013) & 23 \\
\hline & McClelland (1961) & 12.877 \\
\hline & McClelland (1973) & 4.733 \\
\hline & McGrath y Macmillan (2000) & 1.030 \\
\hline & Moriano, Topa, Molero, Entenza y Lévy-Mangin (2012) & 6 \\
\hline & Naktiyok, Karabey y Gulluce (2010) & 23 \\
\hline & Nicolaou y Shane (2014) & 5 \\
\hline & Pihie y Akmaliah (2009) & 79 \\
\hline & Pihie y Bagheri (2010) & 9 \\
\hline & Rae (2006) & 122 \\
\hline & Rauch y Frese (2007) & 522 \\
\hline & Reynolds et al. (2005) & 995 \\
\hline & Schumpeter (1934) & 30.461 \\
\hline & Shane (2003) & 3.012 \\
\hline & Shane y Venkataraman (2000) & 8.268 \\
\hline & Shepherd (2011) & 57 \\
\hline & Stephan, Uhlaner y Stride (2015) & 8 \\
\hline & Stevenson y Jarillo (1990) & 2.624 \\
\hline & Stewart y Roth (2001) & 628 \\
\hline & Stewart y Roth (2007) & 171 \\
\hline & Winterton (2002) & 15 \\
\hline & Yang y Dess (2007) & 7 \\
\hline & Zahra, Korri y Yu (2005) & 319 \\
\hline & Zhao y Seibert (2006) & 624 \\
\hline & Zhao, Seibert y Hills (2005) & 979 \\
\hline & Zhao, Seibert y Hills (2010) & 348 \\
\hline 竞 & Barney (1986) & 6.142 \\
\hline 焉 & North (1990) & 37.998 \\
\hline
\end{tabular}




\begin{tabular}{|c|c|c|}
\hline & Autores & $N^{\circ}$ citas \\
\hline \multirow{36}{*}{ 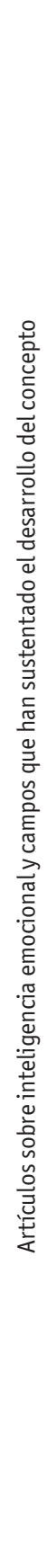 } & Ashkanasy, Becker y Waldman (2014) & 7 \\
\hline & Bar-On, R. (1997) & 888 \\
\hline & Barbey, Colom y Grafman (2012) & 30 \\
\hline & Becker, Cropanzano y Sanfey (2011) & 85 \\
\hline & Boyatzis, Goleman y Rhee (2000) & 907 \\
\hline & Brackett, Rivers y Salovey (2011) & 132 \\
\hline & Carmeli (2003) & 622 \\
\hline & Cartwright y Pappas (2008) & 88 \\
\hline & Chamorro-Premuzic y Furnham (2010) & 57 \\
\hline & Fiori y Antonakis (2011) & 65 \\
\hline & Goleman (1995) & 105 \\
\hline & Goleman (1998) & 7.274 \\
\hline & Goleman y Cherniss (2005) & 6 \\
\hline & Jamali et al. (2008) & 26 \\
\hline & Joseph y Newman (2010) & 475 \\
\hline & Joseph, Jin, Newman y 0'Boyle (2015) & 12 \\
\hline & Killgore et al. (2013) & 7 \\
\hline & Koven, Roth, Garlinghouse, Flashman y Saykin (2011) & 18 \\
\hline & Lee, Senior y Butler (2012) & 27 \\
\hline & Mayer y Salovey (1997) & 617 \\
\hline & Momeni (2009) & 75 \\
\hline & 0'Boyle, Humphrey, Pollack, Hawver y Story (2011) & 259 \\
\hline & Pérez, Petrides y Furnham (2005) & 246 \\
\hline & Petrides y Furnham (2001) & 894 \\
\hline & Rego, Sousa, Cunha, Correia y Amaral (2007) & 81 \\
\hline & Rosete y Ciarrochi (2005) & 450 \\
\hline & Rubin, Munz y Bommer (2005) & 419 \\
\hline & Schnell, Bluschke, Konradt y Walter (2011) & 85 \\
\hline & Senior, Lee y Butler (2011) & 69 \\
\hline & Siegling, Petrides y Martskvishvili (2015) & 4 \\
\hline & Skudiene, Auruskeviciene, Reardon y Stangej (2011) & 3 \\
\hline & Sosa (2008) & 2 \\
\hline & Stubbs, Koman y Wolff (2008) & 137 \\
\hline & Sunindijo, Hadikusumo y Ogunlana (2007) & 94 \\
\hline & Webb et al. (2013) & 18 \\
\hline & Zhou y George (2003) & 374 \\
\hline
\end{tabular}




\begin{tabular}{|c|c|c|}
\hline & Autores & $\mathrm{N}^{\circ}$ citas \\
\hline \multirow{19}{*}{ 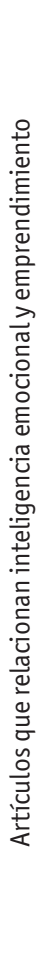 } & Ahmetoglu, Leutner y Chamorro-Premuzic (2011) & 43 \\
\hline & Awwad y Ali (2012) & 1 \\
\hline & Bahadori (2012) & 8 \\
\hline & Cross y Travaglione (2003) & 100 \\
\hline & Dehkordi, Sasani, Fathi y Khanmohammadi (2012) & 4 \\
\hline & Hadizadeh, Raminmehr y Hosseini (2009) & 7 \\
\hline & Karimi, Kloshani y Bakhshizadeh (2012) & 6 \\
\hline & Mortan, Ripoll, Carvalho y Bernal (2014) & 6 \\
\hline & Neghabi, Yousefi y Rezvani (2011) & 7 \\
\hline & Pachulia y Henderson (2009) & 8 \\
\hline & Pradham y Nath (2012) & 5 \\
\hline & Rhee y White (2007) & 51 \\
\hline & Salvador (2008) & 21 \\
\hline & Whitener, Brodt, Korsgaard y Werner (1998) & 1.602 \\
\hline & Wu, McMullen, Neubert y Yi (2008) & 69 \\
\hline & Yitshaki (2012) & 16 \\
\hline & Zakarevičiusy Župerka (2010) & 22 \\
\hline & Zampetakis, Beldekos y Moustakis (2009) & 75 \\
\hline & $\begin{array}{l}\text { Zampetakis, Kafetsios, Bouranta, Dewett y Moustakis } \\
(2009)\end{array}$ & 53 \\
\hline
\end{tabular}

Fuente: elaboración propia.

\section{Fundamentos teóricos}

\subsection{Emprendimiento como campo de estudio}

El emprendimiento representa un campo de estudio de reciente desarrollo que en las últimas tres décadas ha experimentado un importante auge por ser de elevado interés para las sociedades y, por ende, también para los académicos (Low y MacMillan, 2007; Stevenson y Jarillo, 2007). El emprendimiento puede definirse desde una perspectiva genérica como la creación de algo nuevo (Reynolds et al., 2005). Bajo tal perspectiva, la acción de emprender puede implicar o no el desarrollo de una actividad económica (Ahmetoglu et al., 2011) pues no todo nuevo proyecto posee fin lucrativo -ej. fundar un proyecto de voluntariado-. Para aquellos proyectos que conllevan ánimo de lucro, cabe distinguir los que se desarrollan dentro o fuera de los límites de una empresa (Kuratko, 2007) -ej. lanzar al mercado un nuevo producto versus organización de un evento por 
alumnos para reunir fondos para un viaje-. Por ello, la conceptualización que vincula el emprendimiento con la actividad empresarial puede ser considerada restrictiva y limitada (Ahmetoglu et al., 2011).

Martínez-Campos y Cantillo-Guerrero (2013) advierten del uso de diferentes perspectivas epistemológicas en la investigación sobre emprendimiento. De hecho, este campo de estudio se caracteriza por la proliferación de teorías desarrolladas para explicar el fenómeno desde diversas disciplinas científicas como la Economía, la Psicología, la Sociología, la Administración de Empresas o más recientemente la Biología y la Neurociencia. Esta interdisciplinariedad ha generado una dispersión en la investigación realizada en el campo (Kalantantiris, 2004) que, al no haber logrado la integración necesaria, ha dado como resultado la inexistencia de un paradigma unificado (Shane y Venkataraman, 2000). A ello, además, debe sumarse el carácter contextual del emprendimiento, pues los emprendedores operan y son influenciados por su contexto, esto es, por las características socio-económicas de sus entornos (Kalantantiris, 2004).

Dias-Furtado (2015) realiza una exhaustiva revisión de los trabajos hasta el momento publicados en el campo y, basándose en tal revisión, señala que el emprendimiento se ha tratado de explicar, en primer lugar, desde variables que caracterizan a un territorio dado, demostrando la investigación realizada desde un enfoque económico que las estructuras regulativas, normativas y cognitivas en ocasiones incentivan y en otras inhiben el desarrollo del emprendimiento que, en estos trabajos, suele asociarse a la generación de empleo y riqueza en el territorio (North, 1990; Schumpeter, 1934). En segundo lugar, los trabajos desarrollados desde una perspectiva psicológica centran su interés en la persona emprendedora y ponen de manifiesto que la iniciativa empresarial depende de los propios rasgos y atributos individuales (Kuratko, 2007; McClelland 1961; Moriano et al., 2012; Naktiyok et al., 2010). Tales rasgos, en opinión de Dias-Furtado (2015), condicionan las percepciones que las personas tienen de las condiciones socio-económicas que caracterizan un entorno dado. Adicionalmente, y ya desde una perspectiva sociológica, la participación de los individuos en redes asociativas con las que mantienen lazos fuertes y/o débiles puede afectar a su comportamiento emprendedor (Li, Chen, Liu y Peng, 2014). Por último, y en cuanto a los contextos sectoriales de estudio, la literatura ha analizado los factores que incentivan la entrada de los emprendedores principalmente en sectores tales como la alta tecnología (Marvel, 2013), el ámbito rural (Ahmad, Yusoff, Noor y Ramin, 2012) o negocios de carácter social (Iglesias, 2013; Jayawarna, Rouse y Kitching, 2013). 
En este recorrido por las corrientes de investigación existentes para el estudio y la comprensión del emprendimiento merece ser destacado el prolífico cuerpo de literatura desarrollado en el marco del proyecto Global Entrepreneurship monitor, (GEM). Este proyecto, que tiene como misión analizar el papel que juega el emprendimiento en el desarrollo de las economías y en la creación de nuevas empresas (GEM España, 2012) ha dado lugar a numerosos trabajos descriptivos y científicos. Los primeros permiten conocer cómo evoluciona el emprendimiento en los niveles internacional, nacional, y regional y local como áreas diferenciadas dentro de cada país. Los trabajos de naturaleza científica, por su parte, ofrecen las claves para una mayor comprensión de los factores que generan emprendimiento (Cullen, Johnson y Parboteeah, 2014; Stephan, Uhlaner y Stride, 2015). Adicionalmente, la relevancia de los trabajos emanados del GEM viene justificada porque, debido al interés del consorcio por analizar de forma coordinada el emprendimiento en cada lugar, se desarrollan investigaciones que reconocen el carácter contextual del fenómeno y, al tiempo, integran en sus estudios tanto variables relacionadas con el entorno (ej. valores culturales en el territorio, financiación disponible para el emprendimiento, políticas gubernamentales que apoyan el emprendimiento, política fiscal, trabas burocráticas), como con el asociacionismo (ej. integración en redes sociales), o individuales que ayudan a caracterizar al emprendedor (ej. conocimiento, habilidades, experiencia, situación laboral, motivación para emprender, tamaño medio del hogar, etc.) (GEM España, 2012, 2014). Adicionalmente, y dado que se realizan medidas anuales para las variables analizadas, el proyecto ofrece una visión longitudinal y dinámica sobre la evolución en el tiempo del emprendimiento distinguiendo a este respecto las tendencias globales y territoriales de cada lugar analizado. Sin embargo, y a pesar de la globalidad de la información recopilada en el marco del proyecto GEM, los análisis realizados se sostienen en relaciones entre grupos de variables, por lo que persiste la parcialidad como limitación en la búsqueda de la comprensión del emprendimiento (Cullen et al., 2014; Stephan et al., 2015).

Por otra parte, y ya desde el ámbito de la Biología, se ha analizado la predisposición genética a emprender de los individuos, por ejemplo a través de estudios cuantitativos de gemelos (Johnson, 2009) y de individuos adoptados (Lindquist, Sol y Van Praag, 2012), así como la posible influencia de las hormonas y, en particular, de los niveles de testosterona en tal actividad (Guiso y Rustichini, 2011). En lo que a la Neurociencia se refiere, y a pesar de la contribución de esta disciplina a otros ámbitos de la empresa (ej. liderazgo, comportamiento organizativo), hasta el momento no se han realizado investigaciones que aspiren a explicar algún aspecto del emprendimiento (Nicolaou y Shane, 2014). Sin 
embargo, la Neurociencia podría ayudar a los académicos a comprender cómo piensan los emprendedores o por qué reconocen las oportunidades de negocio, o cómo el afecto y la emocionalidad influyen en el comportamiento emprendedor, entre otras cuestiones pendientes de resolver en la literatura (Nicolaou y Shane, 2014).

A pesar de que el emprendimiento, a la luz de la investigación realizada, parece ser un fenómeno solo perceptible y comprensible para los investigadores cuando se analiza a través de dimensiones o perspectivas parciales (Zuluaga, 2010), este, como cualquier otro fenómeno social, constituye un proceso único. Por ello, la interdisciplinariedad del campo corre el riesgo de generar problemas epistemológicos y ontológicos en relación a la multidimensionalidad versus unicidad de los fenómenos sociales (Cassis y Minoglou, 2005). Siendo ello así, cualquier metodología que se emplee para el estudio del emprendimiento (ej. cualitativas, cuantitativas, históricas) debiera plantearse como exigencia epistemológica el situar el tema estudiado dentro de totalidad de las dimensiones y niveles que lo enmarcan (Zuluaga, 2010). Sin embargo, la inexistencia hasta el momento de un paradigma unificado sobre emprendimiento, impide esta deseable orientación de la investigación a acometer (Zuluaga, 2010). En este sentido, la neurocencia puede representar un nuevo paradigma con capacidad para contribuir a la comprensión global del emprendimiento. Sin embargo, y en opinión de Martin y Holan (2014), la resistencia de los académicos a hacer uso de esta nueva disciplina se debe precisamente a la dificultad que entrañan las investigaciones de carácter multidisciplinar, además del coste que representa y la complejidad e innovación que llevan aparejados estos estudios. Como resultado y mientras tanto, los problemas epistemológicos del campo siguen pendientes de resolución.

\subsection{Emprendedor y orientación emprendedora}

El emprendedor puede ser conceptualizado como aquella persona que accede y persigue una idea (Shane y Venkataraman, 2000). Desde la perspectiva más restrictiva, emprendedor sería quien accede a una idea de negocio y organiza los recursos necesarios para aprovecharla, estando dispuesto a asumir un riesgo económico y adoptar un comportamiento proactivo con el propósito de obtener beneficios (Lim, Morse, Mitchell y Seawright, 2010). Con independencia de la conceptualización amplia o estrecha, ha sido común en la literatura recurrir al modelo de intenciones propuesto por Krueger (2005) y distinguir entre la actitud, la intención y el comportamiento emprendedor del individuo. Así, la actitud emprendedora hace referencia a un estado cognitivo o pensamiento favorable al emprendimiento -ej. gusto por la idea de ser emprendedor, considerar ser un empren- 
dedor- (Zampetakis, Kafetsios, Bouranta, Dewett y Moustakis, 2009b). La intención emprendedora, por su parte, alude al compromiso con la acción, esto es, a la voluntad del individuo de ser un emprendedor, de fundar y dirigir un negocio propio -ej. intención de empezar negocio propio en un futuro próximo- (Krueger, 2005). El comportamiento emprendedor se produce cuando el emprendedor de facto organiza los recursos para implantar su idea emprendedora (Shane, 2003). Sobre la base de esta distinción ha sido común en la literatura señalar que la actitud emprendedora precede a la intención (Zampetakis et al., 2009b) y esta, a su vez, al comportamiento emprendedor (Neghabi et al. , 2011).

El desarrollo de nuevos proyectos empresariales tales como la puesta en marcha de un nuevo negocio o la innovación en productos y procesos, pueden ser desarrolladas tanto por individuos independientes como por empresas ya existentes (Neghabi et al., 2011), lo que ha llevado a la literatura a distinguir entre entrepreneurship e intrapreneurship. Para el caso del intrapreneurship o emprendimiento corporativo, son los directivos y líderes emprendedores los que dinamizan estos proyectos en el seno de la empresa y favorecen que esta desarrolle una orientación emprendedora (Moriano et al. , 2012). Tal orientación se erigiría como el conjunto de reglas y normas que guiarían el proceso de toma decisiones en la empresa (Lumpkin y Dess, 1996), materializándose en dimensiones tales como la proactividad, la capacidad para asumir riesgos o la agresividad competitiva (Lumpkin y Dess, 1996).

Para McGrath y Macmillan (2000), los emprendedores se caracterizan porque buscan nuevas oportunidades con perseverancia, poseen una clara orientación a la acción y una capacidad para comprometer sus energías y las de otras personas para lograrlo. Sobre la base de esta descripción, parece razonable pensar que las diferencias entre individuos en lo que a emprendimiento se refiere estén asociadas a diferencias individuales (Stevenson y Jarillo, 1990; Moriano et al., 2012). Por ello, las diferencias en actitud, intención y comportamiento emprendedor de los individuos, ya sean estos empresarios, directivos o líderes, pueden proceder de la existencia de diferencias en personalidad y en aptitudes (Zhao y Seibert, 2006). En este sentido, un constructo candidato para predecir las diferencias individuales en emprendimiento es la inteligencia emocional (IE) (Ahmetoglu et al., 2011; Neghabi et al., 2011; Zakarevičius y Župerka, 2010; Zampetakis et al. , 2009a, 2009b).

\subsection{Inteligencia emocional}

Los procesos cognitivos y emocionales han sido concebidos históricamente de forma separada, es decir, la posible interrelación e interdependencia entre los mismos ha recibido 
escasa atención por parte de los investigadores. Aportando nueva luz en este sentido, en los últimos años la Neurociencia ha hecho considerables avances para una mejor comprensión de la IE integrando ambos tipos de procesos -cognitivos y emocionales-. Así, algunas investigaciones han analizado el procesamiento cognitivo de la emoción centrándose en aspectos tales como el estudio de la empatía cognitiva (Ashkanasy, Becker y Waldman, 2014; Schnell, Bluschke, Konradt y Walter, 2011). Son trabajos que tratan de explicar las bases neuronales de la IE y que, por tanto, nos permiten conocer los mecanismos cognitivos de la regulación emocional (Barbey, Colom y Grafman, 2012). Del mismo modo, existen aproximaciones al estudio de la relación entre el volumen de materia gris y la evaluación de las personas en cuanto a su capacidad para prestar atención a sus estados de ánimo y emociones, su capacidad y claridad para discriminar entre ellos, así como su capacidad para regularlos (Koven, Roth, Garlinghouse, Flashman y Saykin, 2011). Por otra parte, la Neurociencia también ha realizado importantes incursiones en el estudio de la organización (Ashkanasy et al., 2014; Becker, Cropanzano y Sanfey, 2011; Lee, Senior y Butler, 2012; Senior, Lee y Butler, 2011), por lo que podría ofrecer nuevas perspectivas de investigación en relación a la contribución de la IE al éxito de los proyectos empresariales. Parece innegable que una mejor comprensión del cerebro humano puede beneficiar a la ciencia en general y al área de la organización de empresas en particular, en la medida en que se ofrece una base neurocientífica para sustentar el comportamiento organizativo (Senior et al., 2011). La conjugación de los procesos cognitivos y emocionales justifica la atención que ha recibido la IE por parte de la Psicología y de las Ciencias de la Salud, destacando el especial interés que ha tenido su estudio en los ámbitos sociales y empresariales en la última década (Jamali, Sidani y Abu-Zaki, 2008; Zakarevičius y Župerka, 2010).

El hecho de que la IE pueda tener carácter incremental, esto es, que pueda desarrollarse mediante entrenamiento y experiencia (Mayer y Salovey, 1997; Goleman, 1998), justifica un mayor interés en el concepto; las organizaciones pueden beneficiarse de su propia intervención para aumentar las competencias emocionales en los empleados (Cartwright y Pappas, 2008). Por otra parte, la constatación de la validez de la IE más allá de otros constructos tales como la personalidad y la inteligencia puramente cognitiva para explicar el rendimiento en el trabajo consolida aún más dicho interés en este tipo de inteligencia (Joseph y Newman, 2010; 0'Boyle, Humphrey, Pollack, Hawver y Story, 2011). No obstante, esta importancia creciente concedida a la IE no implica, en opinión de Goleman $(1995,1998)$, que se deban desatender las ideas tradicionales asociadas a la inteligencia racional, ya que ésta es también relevante para el individuo y afecta su rendimiento en el trabajo. Es más, si bien se desconoce gran parte de las bases neurobiológicas de la IE, 
según la evidencia la racionalidad estará en el núcleo de los neurocircuítos involucrados en la toma de decisiones emocionales (Killgore et al., 2013).

A pesar del creciente interés en la IE y del grado de acuerdo existente en relación a su relevancia para diferentes ámbitos de la vida, no existe una concepción consensuada del término (Mayer y Salovey, 1997), lo cual contribuye a que las medidas que se proponen para evaluar tal constructo sean también muy diversas (Webb et al., 2013), siendo incluso cuestionados algunos de los instrumentos que se proponen para efectuar dicha medición; como ocurre por ejemplo con los auto-informes (Brackett, Rivers y Salovey, 2011). Lo anterior hace que aún persista el debate en cuanto a la forma de operativizar y medir la IE. No obstante, y aunque la comunidad científica se refiera en muchas ocasiones a la IE como un constructo global, las medidas de IE mejor reconocidas son las que evalúan las diferentes dimensiones de dicha inteligencia (Webb et al., 2013).

Una de las definiciones de IE comúnmente aceptadas es la de Goleman (1998, p. 317) para quien la IE es “la capacidad de reconocer nuestros sentimientos, los sentimientos de los demás, motivarnos y dirigir adecuadamente las relaciones que sostenemos con los demás y con nosotros mismos". Las competencias, por su parte, son aquellos rasgos o conjuntos de hábitos del individuo que le llevan a un desempeño laboral superior (McClelland, 1973). Las diferentes concepciones de la IE y su estructura en competencias emocionales han dado lugar a distintos modelos teóricos que se agrupan en dos tipos: de capacidad o puros y mixtos. Esta distinción reconocida de forma expresa dentro de la investigación científica sobre IE facilita la estructura y el desarrollo de dicho campo de estudio (Siegling, Petrides y Martskvishvili, 2015).

Los modelos de capacidad estudian las habilidades del individuo para procesar información afectiva y consideran la IE como una capacidad cognitiva sustentada en el potencial real de un sujeto para reconocer, procesar y utilizar la información con carga emocional (Ferrando, 2006; Fiori y Antonakis, 2011, Webb et al., 2013). En este tipo de modelos, la IE se considera como una forma de inteligencia pura o conjunto de capacidades cognitivas genéricas que trascienden de un contexto específico (Mayer y Salovey, 1997). Los modelos mixtos o de rasgos tienden a ser más integradores en su conceptualización ya que conjugan dimensiones relativas a la personalidad (Webb et al., 2013) -optimismo, asertividad o empatía- con factores cognitivos y emocionales - percepción, asimilación, comprensión y gestión de las emociones- (Sosa, 2008). En cuanto a su estructura, las competencias emocionales suelen agruparse en cinco áreas principales: autoconocimiento, autorregulación, conciencia social, dirección de relaciones y habilidades sociales (Go- 
leman y Cherniss, 2005). Estos modelos pueden ser considerados como de "autoeficacia emocional" (Pérez, Petrides y Furnham, 2005), ya que relacionan la IE con conductas y capacidades del individuo, tal y como éste las percibe de sí mismo (Petrides y Furnham, 2001). Los modelos mixtos, a diferencia de los de capacidad, trascienden del análisis puramente teórico para buscar un conocimiento práctico y aplicado de la IE (Bar-0n, 1997; Goleman, 1995), en contextos específicos de estudio. Así por ejemplo, estos modelos han servido de base en recientes estudios encaminados a investigar el rendimiento en el trabajo (Joseph y Newman, 2010; Joseph, Jin, Newman y 0’Boyle, 2015).

En la literatura se identifican un modelo de capacidad y diversos modelos mixtos de general aceptación al haber sido utilizados en ulteriores estudios empíricos (Jamali et al., 2008). De ellos destacamos aquí las propuestas de Mayer y Salovey (1997), Goleman $(1995,1998)$ y Bar-On (1997), así como la variante de este último aportada por Petrides y Furnham (2001). El primero se incluye entre los modelos de capacidad, mientras que los de Goleman, Bar-0n y Petrides y Furnham se sitúan en los denominados mixtos. Todos sostienen que la IE otorga a la persona mayor éxito personal y social, y que un individuo con alta inteligencia intelectual no puede operar de manera óptima sin la IE. En nuestra revisión hemos hallado trabajos que relacionan IE y emprendimiento sobre la base de los modelos de capacidad; ej. Neghabi et al. (2011) se sustenta en el modelo de Mayer y Salovey (1997), así como de los modelos mixtos: ej. Rhee y White (2007) o Zakarevičius y Župerka (2010) se basan en la propuesta de Goleman (1998) mientras que Ahmetoglu et al. (2011) o Zampetakis et al. (2009b) en la de Petrides y Furnham (2001). A pesar de esta disparidad en el uso de modelos de IE, el modelo más frecuentemente utilizado en estos estudios es el propuesto por Goleman. Bajo nuestra perspectiva, esto es así porque el modelo de Goleman estudia la IE en el contexto laboral y para directivos (Boyatzis, Goleman y Rhee, 2000). Dicho modelo se estructura en cuatro dimensiones e integra veinte competencias (Boyatzis et al., 2000):

1. Conciencia de uno mismo: autoconciencia emocional, autoevaluación y autoconfianza.

2. Autogestión: autocontrol, fiabilidad, minuciosidad, adaptabilidad, orientación hacia el logro, iniciativa.

3. Conciencia social: empatía, orientación hacia el servicio, conciencia organizativa.

4. Gestión de las relaciones: desarrollar a los demás, influencia, comunicación, resolución de conflictos, liderazgo con visión de futuro, catalizar los cambios, establecer vínculos, trabajo en equipo y colaboración. 


\subsection{La inteligencia emocional como factor explicativo del emprendimiento}

A pesar del creciente interés en la IE como atributo clave para entender el éxito profesional de los individuos en términos de satisfacción, compromiso, implicación y rendimiento en el trabajo (Ahmetoglu et al. , 2011; Carmeli, 2003; 0'Boyle et al. , 2011), liderazgo transformacional eficaz (Rosete y Ciarrochi, 2005; Rubin, Munz y Bommer, 2005) y funcionamiento y rendimiento de los equipos de trabajo (Stubbs y Wolff, 2008), existe muy poca investigación que haya explorado el efecto de este rasgo sobre las diferencias individuales en emprendimiento (Zampetakis et al., 2009a). Debido a ello, en este trabajo realizamos una revisión general contemplando al emprendimiento desde un enfoque amplio. Así, nuestra revisión se ha extendido a aquellos artículos publicados que relacionan ambos conceptos con independencia de la forma en que se conceptualice el emprendimiento, esto es, como una actitud, una intención o un comportamiento del individuo, empresario o no, así como a través de la orientación emprendedora del conjunto de la empresa.

Dado el estado incipiente de esta línea de estudio, la investigación realizada se ha centrado básicamente en analizar si la IE está relacionada con el emprendimiento y/o el éxito emprendedor y, más exiguamente, si esta influencia es independiente y va más allá de la que ejercen los rasgos de personalidad y otras características del individuo (Ahmetoglu et al., 2011), ampliamente investigadas desde la Teoría de rasgos (Kuratko, 2007; Louw et al., 2003). La estructura básica de los trabajos responde al modelo de intenciones propuesto por Krueger (2005), centrándose en la relación IE-actitud emprendedora, IE-intención emprendedora y, en menor medida, IE-comportamiento emprendedor, estando en muchos casos las muestras empíricas integradas por estudiantes (Zakarevičius y Župerka, 2010; Zampetakis et al., 2009b).

\subsection{La influencia directa de la IE en el emprendimiento}

La investigación que relaciona IE y emprendimiento ha estado interesada en justificar mediante argumentos principalmente teóricos si la IE afecta al emprendimiento y/o al éxito emprendedory, más particularmente, qué competencias emocionales del individuo condicionan sus actitudes, intenciones y comportamiento emprendedor. La premisa básica de estos trabajos es que estas competencias de carácter emocional son importantes cualidades del individuo que le ayudan a desarrollar su fortaleza personal y a mejorar su eficacia individual para acometer tareas desafiantes (Man y Lau, 2000). Para el caso del 
emprendedor, tales competencias le ayudarían a afrontar los desafíos que comporta la puesta en marcha y gestión de un negocio, puesto que las mismas parecen relacionarse con la capacidad de la persona para innovar, y para crear y explotar oportunidades (Bahadori, 2012; Man y Lau, 2000).

Con respecto a la relación IE - actitudes e intenciones emprendedoras destacan, entre otros, los trabajos de Neghabi et al. (2011), Zakarevičius y Župerka (2010) y Zampetakis et al. (2009b). Partiendo del hecho de que las actitudes emprendedoras, al igual que todas las actitudes, conllevan información emocional, Zampetakis et al. (2009b) sugieren que los individuos con elevada IE poseen buena percepción sobre su auto-eficacia emocional y menor nivel de aversión al riesgo (Foo, 2011). Estas características emocionales les pueden permitir perseverar en su intención de alcanzar un objetivo cuando surjan problemas imprevistos, siendo esto importante para las actitudes e intenciones relacionadas con la puesta en marcha de una empresa, dado los niveles de riesgo que las actividades empresariales comportan. Por otra parte, Salvador (2008) demuestra que algunas dimensiones de la inteligencia emocional guardan una relación positiva y significativa con la autoeficacia emprendedora, la cual ha sido propuesta en diferentes trabajos de investigación como un antecedente clave de la intención emprendedora (Lee, Wong, Foo, y Leung, 2011; Pihie y Akmaliah, 2009). Esta relación ha sido probada empíricamente para la población estudiantil en trabajos como el de Chen, Greene, y Crick (1998) o Zhao, Seibert y Hills (2005). Los individuos con alta inteligencia emocional creen en sus habilidades emprendedoras y se perciben como personas con más y mejores oportunidades para emprender actividades emprendedoras, puesto que se adaptan mejor al entorno, trabajan mejor bajo condiciones de estrés y se adaptan mejor a los cambios, impulsando todo ello su voluntad emprendedora (Mortan, Ripoll, Carvalho, y Bernal, 2014).

Con respecto al estudio de la influencia de la IE sobre el comportamiento emprendedor, hemos hallado los trabajos de Zampetakis et al. (2009a, 2009b) o Rhee y White (2007). Concretamente, Zampetakis et al. (2009b) afirman que la inteligencia emocional afecta el comportamiento emprendedor a través de dos vías: por una parte, los individuos con una alta autoevaluación en eficacia emocional muestran una mayor tolerancia al estrés $y$, por otra parte, los individuos con alta IE tienden a tener mayor afectividad y una disposición a la proactividad, todo lo cual facilita el comportamiento emprendedor. Rhee y White (2007) afirman que los emprendedores de éxito, entendidos como aquellos que obtienen mayores beneficios se corresponden con individuos con un alto nivel de IE. Esto puede suceder porque el emprendimiento es una actividad fundamentalmente de naturaleza social (Zampetakis et al., 2009b) ya que el emprendedor ha de negociar con 
proveedores y distribuidores para hacer factible la producción y distribución de un producto y la construcción de estas relaciones con los diversos grupos de interés servirá de apoyo a los proyectos emprendidos (Skudiene, Auruskeviciene, Reardon y Stangej, 2011). Por consiguiente, la habilidad para interactuar con otras personas y construir redes de relaciones, rasgo asociado a la IE, es extremadamente importante para la explotación de la oportunidad (Mortan et al., 2014; Chell y Baines, 2000), esto es, para emprender actividades emprendedoras (Salvador, 2008), así como para predecir su éxito (Ahmetoglu et al., 2011; Zampetakis et al., 2009a), tanto para el caso de los negocios de nueva creación como para comprender el comportamiento emprendedor dentro de las organizaciones. Así mismo, la mayor inteligencia emocional del emprendedor anticipa y evita rupturas emocionales, permite dirigir el conflicto, sopesar la información emocional para tomar las mejores decisiones, crear un entorno de trabajo positivo y una alta moral en el equipo (Skudiene et al., 2011). Es por ello que Gerus (2009; en Skudiene et al., 2011) afirmaba, como resultado de su investigación, que entre las diez capacidades clave de la próxima generación de directivos emprendedores destaca, en primer lugar, la inteligencia emocional, que les permitirá alcanzar resultados exitosos en sus proyectos.

La investigación realizada hasta el momento permite, por tanto, avanzar prometedores resultados sobre el efecto de la IE tanto sobre el descubrimiento de la oportunidad como sobre la decisión del individuo de explotar la misma y en la organización de los recursos necesarios para implantar su idea emprendedora, tal y como sugiere Shane (2003) cuando se utiliza la Teoría de rasgos para analizar otros atributos del individuo. A este respecto, podemos coincidir con Shane y Venkataraman (2000) cuando afirman que la IE aporta un nuevo enfoque para la comprensión del proceso a través del cual los emprendedores reconocen y desarrollan nuevas oportunidades, demostrando por tanto ser un constructo clave al determinar el emprendimiento (Dehkordi, Sasani, Fathi y Khanmohammadi, 2012).

Ahora bien, la IE se estructura en competencias emocionales que permiten al individuo actuar sobresalientemente y obtener buenos resultados en los proyectos que acomete (Boyatzis et al., 2000; Goleman, 1998; Mayer y Salovey, 1997). Esta idea ha sido testada por investigadores del campo y sus trabajos dejan constancia de que las competencias relacionadas con la autogestión (ej. iniciativa) o con la dirección de las relaciones (ej. liderazgo con visión de futuro, influencia, colaboración) generan estos resultados superiores (Goleman, 1995; Rhee y White, 2007). Para el caso del emprendimiento, Winterton (2002) propuso modelos multidimensionales de competencias necesarias para que el directivo de una PYME desempeñara la función emprendedora y en su modelo incluyó competencias relacionadas con la conciencia de uno mismo (autoconfianza), la autoges- 
tión (autocontrol, orientación al logro) y la dirección de las relaciones (comunicación, capacidad para construir equipos de trabajo, persuasión). Asimismo, los emprendedores participantes en las investigaciones realizadas por Awwad y Ali (2012), Nezhad, Marjani y Najafi (2015), Pradham y Nath (2012) y Rhee y White (2007) demostraron tener mayores niveles en todas las categorías de competencias, esto es, en las relacionadas con la conciencia de uno mismo (autoconfianza), la autogestión (fiabilidad, orientación al logro), la conciencia social (orientación hacia el servicio, empatía) y la gestión de las relaciones (catalizar los cambios, trabajo en equipo y colaboración). De esta forma y opuestamente a la idea tradicional de que los emprendedores son individualistas y autónomos (McClelland, 1961), se encuentra que estos poseen altos niveles en competencias relacionadas con el trabajo en grupo y la colaboración (Rhee y White, 2007).

A pesar de esta coincidencia general sobre la importancia de las competencias emocionales para el emprendimiento, algunos autores han hallado resultados en cierta medida discordantes y que por tanto merecen ser comentados. Así, Ahmad, Abdul Halim y Mohamed Zainal (2010) analizan las competencias emocionales asociadas al rol de emprendedor en una PYME e identifican las siguientes: creer en uno mismo, autoconocimiento, autocontrol y tolerancia al estrés, automotivación y capacidad de dirigirse a sí mismo, todas relacionadas con la conciencia de uno mismo y la autogestión, es decir, con el propio individuo. Por su parte, Dehkordi et al. (2012) en su trabajo clasifican diversas competencias emocionales en función de su relevancia para la intención emprendedora. Para estos autores, la autogestión y la conciencia social eran las más importantes mientras que el autoconocimiento y la dirección de las relaciones tenían un menor impacto. Por su parte, Karimi, Kloshani y Bakhshizadeh (2012) encuentran que entre las principales competencias emocionales que diferencian a los emprendedores más y menos exitosos destacan las relaciones interpersonales, la autoconciencia y la conciencia social (empatía).

Bajo nuestro punto de vista sorprende que, a pesar de ser el emprendimiento una actividad de naturaleza social (Zampetakis et al., 2009b) debido a la interacción permanente que el emprendedor ha de mantener con otros actores económicos y sociales, así como con sus empleados, los trabajos de Ahmad et al. (2010) y Dehkodi et al. (2012) no identifiquen en el catálogo de competencias emocionales para el emprendimiento las relacionadas con la dirección de las relaciones (ej. influencia, comunicación, resolución de conflictos, liderazgo con visión de futuro, establecer vínculos, trabajo en equipo y colaboración). Entendemos que el hecho de que Dehkodi et al. (2012) analicen la intención emprendedora y no el comportamiento emprendedor ni sus resultados puede justificar estos hallazgos. Según el modelo de Krueger (2005), la intención emprendedora precede a la 
acción, por lo que el emprendedor en intención aún no ha asumido el reto de afrontar la interacción real con su entorno para explotar su oportunidad fundando el proyecto. Bajo nuestro punto de vista, esto podría explicar que la intención emprendedora, aun siendo un predictor del comportamiento emprendedor, no explique este fenómeno en su totalidad pues, es posible que muchos individuos con intención emprendedora carezcan de competencias emocionales para la dirección de las relaciones que resultarán fundamentales para acometer con éxito la acción emprendedora.

Por otra parte, algunos trabajos desarrollados en contextos muy específicos han encontrado que la IE no es relevante para aquellos que pretenden generar una orientación emprendedora en sus empresas (Pachulia y Henderson, 2009). Estos resultados inesperados o contradictorios aconsejan continuar indagando en el carácter de la relación IE-emprendimiento.

Los argumentos anteriores nos permiten plantear las siguientes proposiciones de investigación:

P1: La IE del individuo influye de forma directa positiva y significativa en su actitud, intención y comportamiento emprendedor.

P1-1: Las competencias emocionales relacionadas con la conciencia de uno mismo, la autogestión y la conciencia social influirán de forma positiva en la conformación de la actitud e intención emprendedora.

P1-2: Las competencias emocionales relacionadas con la conciencia de uno mismo, la autogestión, la conciencia social y la gestión de las relaciones influirán de forma positiva en el comportamiento emprendedor.

P2: La IE del individuo influye de forma directa positiva y significativa en el éxito de su proyecto emprendedor.

\subsection{La influencia indirecta de la IE en el emprendimiento}

La revisión de la literatura muestra que la IE del individuo puede afectar la intención o la acción emprendedora de forma indirecta a través de su influencia en una serie de factores de naturaleza personal y organizativa que han mostrado ser determinantes del emprendimiento en sus diferentes facetas. Así, Chamorro-Premuzic y Furnham (2010) establecen que sería interesante demostrar la validez incremental de la IE frente a otros factores de naturaleza personal a la hora de explicar las intenciones o comportamientos 
del individuo. En este sentido, Ahmetoglu et al., (2011) demuestran que, efectivamente, la IE tiene una validez incremental en la predicción de algunas actividades emprendedoras más allá del carácter predictivo de la personalidad y otras variables demográficas.

La IE podría ser un antecedente indirecto de la actitud e intención emprendedoras al estar su influencia mediada por factores identificados como facilitadores del emprendimiento, esto es, la capacidad innovadora (Kuratko, 2007), la proactividad, y la propensión a asumir riesgos. Así, Sunindijo, Hadikusumo y Ogunlana (2007), Zampetakis et al. (2009b) o Zhou y George (2003) establecen que la IE como indicador de autoeficacia emocional antecede a la actitud e intención emprendedora a través de la disposición del individuo a ser creativo y proactivo. Enlazando estos hallazgos con la innovación como variable clave para el emprendimiento, Kuratko (2007) y Ahmetoglu et al. (2011) consideran que los individuos con una alta IE tendrán mayor disposición a la creatividad facilitando así la innovación, lo cual representa un avance para la comprensión del emprendimiento. Desde un enfoque de resultados, Sunindijo et al. (2007) establecen que los directores de nuevos proyectos con una elevada IE tenderán a utilizar la comunicación abierta y un estilo de liderazgo proactivo que pueden proporcionar resultados positivos. La influencia de la IE en el crecimiento de los proyectos emprendidos es indirecta, y se ejerce a través de la habilidad del emprendedor de guiar a sus seguidores en un entorno dinámico y lleno de incertidumbre en relación tanto a los productos como al mercado (Ensley, Pearce y Hmieleski, 2006) permitiéndoles incluso contribuir a la toma de decisiones mostrando confianza en ellos (Whitener, Brodt, Korsgaard y Werner, 1998). Por tanto, todas estas variables serían antecedentes de las habilidades directivas necesarias para que un nuevo proyecto emprendido crezca y tenga éxito (Yitshaki, 2012).

Con base en lo anterior, podemos plantear que los directivos emocionalmente inteligentes pueden impulsar la voluntad de los empleados de actuar de forma emprendedora (Brundin, Patzelt y Shepherd, 2008), voluntad que se verá reforzada por la propia IE del empleado que avivará su voluntad de innovar (Hadizadeh, Raminmehr y Hosseini, 2009). Esto último sucede porque los empleados con alta IE son más conscientes de aquellos factores que contribuyen a que ellos mismos experimenten emociones positivas y negativas, de modo que las acciones emprendedoras podrían producirse debido a las percepciones del empleado acerca de sus propias habilidades emocionales que le permiten experimentar más confianza y control sobre los requisitos del entorno de trabajo y por ende actuar de forma emprendedora (Zampetakis et al., 2009b). Del mismo modo, Rego, Sousa, Cunha, Correia y Amaral (2007), Zampetakis et al. (2009b) y Zhou y George (2003) observan que aquellos directivos con un alto grado de IE pueden incentivar la creatividad de los 
empleados, lo cual conducirá a una alta orientación emprendedora de la empresa en la medida en que se generan nuevas ideas que a su vez permiten explorar nuevos modelos de negocio (Awwad y Ali, 2012; Wu, McMullen, Neubert y Yi, 2008). Sin embargo, una inteligencia emocional pobre en un líder tiende a reflejarse de forma negativa en la creatividad de los empleados (Rego, et al., 2007). En palabras de Momeni (2009) y Zampetakis et al. (2009b) la IE conseguirá crear un clima organizativo que mediará el efecto que ejerce la IE del directivo en la orientación emprendedora de la empresa. Esta relación fue testada por Awwad y Ali (2012), quienes encontraron que la IE del directivo pretende crear un clima caracterizado por el apoyo directivo, la claridad de roles de los empleados, y la sensación de los empleados de que pueden contribuir a las metas de la organización. A su vez se muestra que todo ello refuerza la creatividad de los empleados y, por ende, la orientación emprendedora.

En cuanto a los rasgos personales, la investigación de Pradham y Nath (2012) muestra que la IE está relacionada de forma positiva y significativa con la orientación al logro, el locus de control, la propensión a asumir riesgos, la autoconfianza, la extroversión y la capacidad para establecer redes asociativas. Estas variables contribuyen a definir la personalidad del individuo y dichos elementos de la personalidad han mostrado contribuir sustancialmente al modo en que piensan los emprendedores, a los objetivos que persiguen, las acciones que emprenden y a sus logros (Brandstätter, 2011; Mortan et al., 2014; Rauch y Frese, 2007, Stewart y Roth, 2001, 2007; Zhao y Seibert, 2006; Zhao et al. , 2005; y Zhao, Seibert y Lumpkin, 2010). Por tanto, la IE puede contribuir, a través de dichas variables de personalidad, a predecir el emprendimiento y su éxito. Otro rasgo personal que muestra relación con la IE es la capacidad de percepción del individuo. Así, Pradham y Nath (2012) encontraron que la IE está relacionada de forma positiva y significativa con la percepción de oportunidades de negocio, contribuyendo de este modo a la intención emprendedora.

Centrando su atención en las dinámicas de comportamiento entre las personas, Rhee y White (2007) encontraron que la confianza que inspira el emprendedor es su principal competencia. Así, dicha confianza, que incorpora elementos de tipo cognitivo (competencia e integridad) y afectivo (benevolencia), podría concebirse como uno de los ingredientes del emprendimiento exitoso. Según Goleman (1998), una persona en la que se puede confiar actúa éticamente, construye confianza a partir de su fiabilidad, admite sus propios errores y adopta una postura difícil y basada en sus principios a pesar de que ello sea impopular. Es probable que sin generar confianza el emprendedor tenga dificultades para construir la red de apoyo necesaria para asegurar el éxito de su proyecto de creación 
de empresa (Rhee y White, 2007). Todo ello nos indica una influencia indirecta de la IE sobre el comportamiento emprendedor en la medida en que la IE de las personas influye en sus dinámicas de comportamiento (ej. confianza, conflicto).

Los planteamientos expuestos acerca de la influencia indirecta de la IE en el emprendimiento pueden sintetizarse en las siguientes proposiciones:

P3: La IE del individuo influye de forma indirecta en el emprendimiento a través de los factores facilitadores del mismo:

P3-1: La IE influye de forma indirecta en el emprendimiento a través de la creatividad y proactividad del emprendedor.

P3-2: La IE influye de forma indirecta en el emprendimiento a través de la propensión a la innovación del emprendedor.

P3-3: La IE influye de forma indirecta en el emprendimiento a través de la propensión a asumir riesgos del emprendedor.

P4: La IE del individuo influye de forma indirecta en el emprendimiento a través de los rasgos de personalidad del emprendedor, así como de sus percepciones.

P5: La IE del individuo influye de forma indirecta en el emprendimiento a través la confianza que dicho emprendedor sea capaz de inspirar.

\section{Discusión}

Dada la relevancia del emprendimiento para el crecimiento económico y la generación de riqueza en un territorio, el estudio de los factores que condicionan el mismo es de gran interés (Yang y Dess, 2007). Sin embargo, la investigación hasta el momento realizada y fundamentada en distintos enfoques teóricos -ej. Teoría económica, Visión basada en los recursos, Teoría de rasgos- si bien ha conseguido identificar múltiples antecedentes que propician la acción emprendedora no logran explicar en su totalidad por qué unos individuos emprenden y otros no, cuestión de máxima importancia para orientar los esfuerzos que los actores económicos y políticos desarrollan para impulsar el comportamiento emprendedor en sus territorios.

Teniendo en cuenta que la decisión emprendedora y la forma que el individuo da a su proyecto empresarial viene determinada por criterios racionales y no racionales (Zahra et al., 2005), nuestro trabajo acude a la literatura sobre IE como alternativa para expli- 
car por qué ciertos individuos emprenden. Esta decisión la adoptamos porque existe un cuerpo creciente de literatura que avala la relevancia de la IE para explicar tanto el éxito profesional de los individuos (0'Boyle et al., 2011) como las diferencias individuales en emprendimiento (Zampetakis et al., 2009b), tal y como evidencia la revisión teórica llevada a cabo y cuya discusión nos ha permitido formular diferentes proposiciones de investigación. A pesar de ello, los trabajos interesados en conocer la relación IE-emprendimiento son considerados escasos por autores como Ahmetoglu et al. (2011) y Neghabi et al. (2011). Nosotros creemos, además, que la literatura que aborda el estudio de esta relación debe ser calificada como dispersa, fragmentada, insuficiente y, en algunos casos, mixta o contradictoria, reflejando el estado incipiente en el que se encuentra esta línea de estudio.

El carácter disperso y fragmentado viene probablemente propiciado por la existencia de múltiples conceptualizaciones del emprendimiento y de modelos para el estudio de la IE. El emprendimiento puede ser analizado desde el ángulo del individuo, y atendiendo a su actitud, intención y/o comportamiento emprendedor, dentro o fuera de la empresa, pero también referido a la actuación del conjunto de la empresa. Lógicamente y dado que los factores que subyacen a la actitud o intención emprendedora, constructos de naturaleza fundamentalmente cognitiva, y al comportamiento emprendedor, más relacionado con la acción, son diferentes, el uso de diferentes conceptualizaciones del emprendimiento puede afectar los resultados hallados en la relación IE-emprendimiento. Por su parte, la IE puede ser analizada desde un enfoque meramente cognitivo o de inteligencia pura o mediante modelos mixtos que incluyen en sus medidas elementos cognitivos y no cognitivos tales como ciertos rasgos de la personalidad del individuo. Lógicamente, la utilización de diferentes modelos de IE también puede afectar los resultados. De esta forma, la inexistencia de trabajos globales que incorporen en un único modelo teórico las formas en que la IE afecta a los diferentes constructos que permiten medir el emprendimiento limita la comprensión del fenómeno. Por ello, el desarrollo de la literatura podría beneficiarse de los esfuerzos por construir dicho modelo global o unificado.

La investigación que relaciona IE y emprendimiento debe ser calificada además de insuficiente en lo que se refiere al alcance de los objetivos de investigación y pretensión de los trabajos empíricos. En efecto, buena parte de los artículos publicados han estado interesados en determinar y justificar mediante argumentos teóricos cómo o por qué la IE afecta al emprendimiento y/o al éxito emprendedor y, más particularmente, qué competencias emocionales específicas del individuo condicionan sus actitudes, intenciones y comportamiento emprendedor. Sin embargo, los estudios empíricos son escasos y, en 
muchos casos, sustentados en muestras de estudios conformadas por estudiantes. Si bien estos trabajos pueden realizar una aproximación preliminar sobre la relación objeto de estudio, tienen una capacidad limitada para contribuir a la comprensión real de la acción emprendedora que, más allá de las actitudes e intenciones, representa el verdadero motor de la innovación, empleo y riqueza en un territorio. Entendemos que esto es así porque, a pesar de la importancia que tiene comprender cómo se genera la actitud o la intención emprendedoras, al preceder estas a la acción emprendedora, no siempre dichas actitudes o intenciones se materializan en acciones, siendo necesario que la investigación avance hasta el estadio final del modelo de intenciones propuesto por Krueger (2005).

Adicionalmente, nuestra revisión de la literatura sólo ha hallado estudios empíricos sustentados en autoinformes a través de los cuales los individuos evalúan sus propias competencias emocionales, a pesar de las recomendaciones realizadas por los investigadores del campo de la IE sobre la idoneidad de los estudios basados en técnicas de $180^{\circ}$ y $360^{\circ}$ debido a su carácter más objetivo (Ahmetoglu et al., 2011). Además, todos los estudios revisados son de naturaleza transversal por lo que no permiten analizar el orden causal existente entre IE y emprendimiento ni hacer predicciones sobre el progreso de la IE, variable de carácter incremental (Mayer y Salovey, 1997; Goleman, 1998) que puede mejorar a medida que el individuo aprende de sus experiencias como emprendedor. Por consiguiente, para el progreso de esta línea de investigación deben desarrollarse investigaciones empíricas sustentadas en diseños longitudinales y basadas en técnicas de medición de la IE trianguladas y, por tanto, más objetivas y fiables.

Finalmente, la investigación IE-emprendimiento puede ser calificada como mixta o contradictoria dado que los resultados alcanzados no siempre son coherentes o apuntan en la dirección esperada. Así, y a pesar de la coincidencia general existente sobre la importancia de las diferentes competencias emocionales para el emprendimiento, algunos autores han hallado que algunas de estas competencias, tales como las relacionadas con la dirección de las relaciones sociales (comunicación, resolución de conflictos, trabajo en equipo, colaboración), tienen un menor impacto sobre el emprendimiento (Ahmad et al., 2010; Dehkordi et al., 2012). Dada la naturaleza social de la actividad emprendedora (Zampetakis et al., 2009b), que requiere de una interacción permanente del emprendedor con otros actores, tales resultados sorprenden por inesperados. Por otra parte, algunos trabajos desarrollados en contextos muy específicos han encontrado que la IE no es relevante para los directivos o emprendedores en su función de generar una orientación emprendedora en sus organizaciones (Pachulia y Henderson, 2009). Finalmente, la revisión realizada no nos ha permitido concluir si la posible influencia de la IE sobre el 
emprendimiento es directa y va más allá de la que ejercen los rasgos de personalidad y otras características demográficas del individuo (Ahmetoglu et al., 2011) o si es indirecta y afecta al emprendimiento sólo en la medida que determina el desarrollo de ciertos rasgos de la personalidad de éste (Neghabi et al., 2011); es más algunos estudios apuntan una posible relación recíproca según la cual IE y personalidad se influyen mutuamente para finalmente condicionar la actividad emprendedora del individuo (Zakarevičius y Župerka, 2010). Todo este conjunto de resultados aconsejan continuar indagando en el carácter de la relación IE-emprendimiento aquí analizada.

Ahora bien, las limitaciones hasta el momento apuntadas en la investigación IE-emprendimiento y que corroboran el carácter incipiente de esta línea de estudio, no excluyen que autores como Awwad y Ali (2012) o Shepherd (2011) mantengan grandes expectativas sobre lo que la línea puede aportar al campo. En efecto, los resultados hasta el momento hallados permiten aventurar un efecto positivo de la IE sobre el descubrimiento de la oportunidad y sobre la decisión del individuo de explotarla, indicios suficientes para invitar a profundizar en cuestiones relevantes para el campo tales como: ¿La IE del individuo influye en la motivación que da lugar al emprendimiento, esto es, la búsqueda de la oportunidad y la riqueza -ej.:, emprendimiento por oportunidad-frente a la búsqueda de un medio de vida alternativo dada la imposibilidad de emplearse por cuenta ajena -ej.:, emprendimiento por necesidad-? ¿Los factores contextuales del entorno -ej. existencia de ayudas- afectan de manera similar a individuos con alta o con baja IE, o son aquellos de menor IE los que precisan de un entorno más favorable para tomar la decisión de emprender? ¿Cómo afecta la IE a la decisión de un individuo de fundar un nuevo negocio tras una experiencia previa fallida?

La respuesta a estas cuestiones y el progreso en la investigación realizada puede ser especialmente útil para el desarrollo económico de un territorio. Así, conocer si la IE afecta a la actitud, a la intención y al comportamiento emprendedor, e identificar las competencias que condicionan cada uno de estos constructos, puede ser fundamental para orientar los esfuerzos que desde los ámbitos económicos, políticos y educativos se realizan para impulsar el emprendimiento.

En lo que a las organizaciones se refiere, la investigación sobre IE-emprendimiento también puede ser útil porque ayudaría a comprender qué competencias emocionales deben estimularse en directivos y empleados cuando se desea que la organización se comporte de forma emprendedora, impulsando el desarrollo de innovaciones y nuevos proyectos que permitan su mejor posicionamiento en el mercado. Dado el carácter incremental de 
la IE, la formación en competencias emocionales podría beneficiar y mejorar los niveles de IE de estos individuos y, consecuentemente, contribuir a la creación de una cultura emprendedora en la empresa (Awwad y Ali, 2012).

\section{Conclusiones}

El presente trabajo de investigación se ha desarrollado con el propósito de conocer la relación existente entre la IE del individuo y su comportamiento emprendedor, tratando de discernir si se trata de una relación directa o indirecta, es decir, a través de otros factores organizativos o personales que vienen influenciados por la IE para, a partir de este análisis, formular proposiciones de investigación que ofrezcan posibles líneas de investigación al campo. Con el propósito de responder a este objetivo se ha llevado a cabo una amplia revisión de la literatura publicada sobre IE, sobre emprendimiento y sobre la relación potencialmente existente entre ambos constructos.

Sobre la base del análisis realizado de los marcos teóricos y trabajos empíricos presentados en estas publicaciones podemos sugerir tanto la influencia directa y positiva de la IE sobre la actitud, intención y comportamiento emprendedor del individuo como la influencia indirecta de la misma a través de diversos factores facilitadores del emprendimiento (ej. creatividad y proactividad del emprendedor, su propensión a la innovación o su preferencia a la asunción de riesgos), a través de los rasgos de la personalidad del emprendedor y a través de la confianza que el emprendedor sea capaz de generar en otros. Los estudios analizados nos han permitido discernir, pero no concluir, si la influencia potencial de la IE sobre el emprendimiento es de naturaleza directa o indirecta. Como alternativa, nuestro trabajo propone un conjunto de cinco proposiciones de investigación y otras cinco sub-proposiciones que reflejan las posibles relaciones existentes con el propósito de plantear posibles líneas de investigación al campo de forma que, de llevarse a cabo las pertinentes investigaciones para contrastar las mismas, se podría dilucidar el alcance y la forma en que la IE afecta al emprendimiento.

Adicionalmente, y como resultado de nuestra indagación en la literatura previa, hemos hallado ciertas características que son reveladoras del estado incipiente en que se encuentra esta línea de estudio. En concreto, la investigación es escasa, dispersa, fragmentada, insuficiente y, en algunos casos, mixta o contradictoria. Al objeto de subsanar estas limitaciones y alcanzar resultados concluyentes la investigación posterior que se realice podría considerar ciertas sugerencias de carácter teórico y metodológico también formuladas en el presente trabajo. Entre ellas destacan la necesidad de extender las inves- 
tigaciones en su alcance teórico (ej. actitud, intención y comportamiento emprendedor, elementos cognitivos y no cognitivos de la IE) de forma que sea posible desarrollar un modelo explicativo global y unificado del fenómeno objeto de estudio. Desde un punto de vista metodológico, la utilización de muestras conformadas por emprendedores y no emprendedores, la inclusión de medidas de la IE que superen las limitaciones asociadas al auto-informe del individuo, así como el recurrir a diseños longitudinales que permitan analizar y concluir la existencia de un efecto causal IE-emprendimiento, o viceversa, parece necesario dado la naturaleza incremental de la IE y la posibilidad de que ésta mejore como parte del proceso de aprendizaje que afronta el individuo a través de sus experiencias empresariales.

El esfuerzo investigador realizado en los últimos años en el ámbito de la IE y el emprendimiento es realmente significativo (tómese como referencia que el 43,6\% de los 117 trabajos revisados en el presente artículo han sido publicados en el período 2010-2015), lo que revela la importancia actual que la academia está otorgando a estos constructos. Para terminar, el presente artículo ha pretendido realizar una pequeña aportación al sugerir, a raíz de las aportaciones de la investigación previa, posibles líneas de trabajo que podrían contribuir al desarrollo de este campo de estudio.

\section{Referencias}

Ahmad, A.R., Yusoff, W.F.W., Noor, H.M., and Ramin, A.K. (2012). Preliminary study of rural entrepreneurship development program in Malaysia. Journal of global entrepreneurship, $2(1), 1-8$.

Ahmad, N.H., Abdul Halim, H., and Mohamed Zainal, S.R. (2010). Is entrepreneurial competency the silver bullet for SME Success in a developing nation? Interdisciplinary Journal of Contemporary Research in Business, 2 (1), 217-236.

Ahmetoglu, G., Leutner, F., and Chamorro-Premuzic, T. (2011). EQ-nomics: Understanding the relationship between individual differences in Trait Emotional Intelligence and entrepreneurship. Personality and Individual Differences, 51 (8), 1028-1033.

Ashkanasy, N.M., Becker, W.J., and Waldman, D.A. (2014). Neuroscience and organizational behavior: Avoiding both neuro-euphoria and neuro-phobia. Journal of Organizational Behavior, 35 (7), 909-919.

Awwad, M.H., and Ali, H. (2012). Emotional intelligence and entrepreneurial orientation: The moderating role of organizational climate and employees' creativity. Journal of Research in Marketing and Entrepreneurship, 14 (1), 115-136. 
Bahadori, M. (2012). The effect of emotional intelligence on entrepreneurial behavior: A case study in a medical science university. Asian Journal of Business Management, 4 (1), 81-85.

Barbey, A. K., Colom, R., and Grafman, J. (2012). Distributed neural system for emotional intelligence revealed by lesion mapping. Social cognitive and affective neuroscience, nss124.

Barney, J.B. (1986). Strategic factor markets: Expectations, luck, and business strategy. Management Science, 32 (10), 1231-1241.

Bar-0n, R. (1997). The Bar-On emotional quotient inventory (EQ-I): A test of emotional intelligence. Toronto: Multi-Health Systems.

Becker, W. J., Cropanzano, R., and Sanfey, A. G. (2011). Organizational neuroscience: Taking organizational theory inside the neural black box. Journal of Management, 37, 933-961.

Boyatzis, R.E., Goleman, D., and Rhee, K. (2000). Clustering competence in emotional intelligence: Insights from the emotional competence inventory (ECI). In R. Bar-0n, J.D.A. Parker (eds). Handbook ofemotional intelligence (pp. 343-362). San Francisco: Jossey- Bass.

Brackett, M. A., Rivers, S. E., and Salovey, P. (2011). Emotional intelligence: Implications for personal, social, academic, and workplace success. Social and Personality Psychology Compass, 5 (1), 88-103.

Brandstätter, H. (2011). Personality aspects of entrepreneurship: A look at five meta-analyses. Personality and Individual Differences, 51 (3), 222-230.

Brundin, E., Patzelt H., and Shepherd D.A. (2008). Managers' emotion displays and employees' willingness to act entrepreneurially. Journal of Business Venturing, 23 (2), 221-243.

Carmeli, A. (2003). The relationship between emotional intelligence and work attitudes, behavior and outcomes: An examination among senior managers. Journal of Managerial Psychology, 18 (8), 788-813.

Cartwright, S., and Pappas, C. (2008). Emotional intelligence, its measurement and implications for the workplace. Journal of Management Reviews, 10 (2), 149-171.

Cassis, Y., and Minoglou, I.P. (2005). Entrepreneurship in theory and history: State of the art and new perspectives. In Cassis Y., and I. P. Minoglou (ed.), Entrepreneurship in theory and history (pp. 3-21). Houndmills, Basingstoke, Hampshire, UK: Palgrave Macmillan.

Chamorro-Premuzic, T., and Furnham, A. (2010). The psychology of personnel selection. Cambridge: Cambridge University Press.

Chell, E., and Baines, S. (2000). Networking, entrepreneurship and microbusiness behavior. Entrepreneurship \& Regional Development, 12 (3), 195-215.

Chen, C. C., Greene, P. G., and Crick, A. (1998). Does entrepreneurial self-efficacy distinguish entrepreneurs from managers? Journal of Business Venturing, 13 (4), 295-316.

Cross, B., and Travaglione, A. (2003). The untold story: Is the entrepreneur of the 21st century defined by emotional intelligence? The International Journal of Organizational Analysis, $11(3), 221-228$. 
Cullen, J.B., Johnson, J.L., and Parboteeah, K.P. (2014). National rates of opportunity entrepreneurship activity: Insights from institutional anomie theory. Entrepreneurship Theory and Practice, 38, 775-806.

Dehkordi, A.M., Sasani, A., Fathi, M.R., and Khanmohammadi, E. (2012). Investigating the effect of emotional intelligence and personality traits on entrepreneurial intention using the fuzzy Dematel method. International Journal of Business and Social Science, 3 (13), 286-296.

Dias-Furtado, J. (2015). Instituciones y percepciones institucionales como antecedentes del emprendimiento en economías en desarrollo: una aplicación al sector turístico. Tesis doctoral, Universidad de Las Palmas de Gran Canaria.

Ensley, M. D., Pearce, C. L., and Hmieleski, K. M. (2006). The moderating effect of environmental dynamism on the relationship between entrepreneur leadership behavior and new venture performance. Journal of Business Venturing, 21 (2), 243-263.

Ferrando, M. (2006). Creatividad e inteligencia emocional: un estudio empírico en alumnos con altas habilidades. Tesis doctoral, Universidad de Murcia.

Fiori, M., and Antonakis, J. (2011). The ability model of emotional intelligence: Searching for valid measures. Personality and individual differences, 50 (3), 329-334.

Foo, M.D. (2011). Emotions and entrepreneurial opportunity evaluation. Entrepreneurship Theory and Practice, 35 (2), 375-393.

GEM España (2012). Global Entrepreneurship Monitor. Informe GEM España 2012. Santander, España: Cise, Fundación Xavier de Salas.

GEM España (2014). Global Entrepreneurship Monitor. Informe GEM España 2014. Santander, España: Editorial de la Universidad de Cantabria.

Goleman, D. (1995). Emotional Intelligence. Why it can matter more than IQ?. New York: Bantam.

Goleman, D. (1998). Working with emotional intelligence. New York: Bantam.

Goleman, D., and Cherniss, C. (2005). Inteligencia emocional en el trabajo. Barcelona: Ed. Kairos.

Guiso, L., and Rustichini, A. (2011). Understanding the size and profitability of firms: The role of a biological factor (European University Institute Working Paper ECO 2011-01). Retrieved from http://cadmus.eui.eu/handle/1814/15642

Hadizadeh, M.A., Raminmehr, H., and Hosseini, S.S. (2009). Relationship between emotional intelligence and entrepreneurial orientation among the employees of oil company-affiliated organizations. Journal of Entrepreneurship Development, 1 (4), 139-162.

Iglesias, A. (2013). La persona emprendedora social: desarrollo de un modelo de generación de intenciones socialmente emprendedoras en la Comunidad Autónoma de Euskadi. Tesis doctoral, Universidad de Deusto.

Jamali, D., Sidani, Y., and Abu-Zaki, D. (2008). Emotional intelligence and management development implications. Journal of Management Development, 27 (3), 348-360. 
Jayawarna, D., Rouse, J., and Kitching, J. (2013). Entrepreneur motivations and life course. International Small Business Journal, 31 (1), 34-56.

Johnson, W. (2009). So what or so everything? Bringing behavior genetics to entrepreneurship research. Journal of Business Venturing, 24, 23-26.

Joseph, D. L., and Newman, D. A. (2010). Emotional intelligence: An integrative meta-analysis and cascading model. Journal of Applied Psychology, 95, 54-78.

Joseph, D. L., Jin, J., Newman, D. A., and O'Boyle, E. H. (2015). Why does self-reported emotional intelligence predict job performance? A meta-analytic investigation of mixed EI. The Journal of applied psychology, 100 (2), 298.

Kalantaridis, C. (2004). Understanding the entrepreneur, an institutionalist perspective. Aldershot, Hants, UK: Ashgate.

Karimi, P., Kloshani, M., and Bakhshizadeh, A. (2012). A comparative study on emotional intelligence and cognitive between successful and unsuccessful entrepreneurs. Management Science Letters, 2 (6), 2071-2076.

Killgore, W. D., Schwab, Z. J., Tkachenko, 0., Webb, C. A., et al. (2013). Emotional intelligence correlates with functional responses to dynamic changes in facial trustworthiness. Social neuroscience, 8 (4), 334-346.

Koven, N. S., Roth, R. M., Garlinghouse, M. A., Flashman, L. A., and Saykin, A. J. (2011). Regional gray matter correlates of perceived emotional intelligence. Social cognitive and affective neuroscience, 6 (5), 582-590.

Krueger, N. F. (2005). The cognitive psychology of entrepreneurship. En Handbook of entrepreneurship research, 1 (pp. 105-140). US: Springer.

Kuratko, D. F. (2007). Entrepreneurial leadership in the 21stcentury: Guest editor's perspective. Journal of Leadership and Organisational Studies, 13 (4), 1-11.

Lee, N., Senior, C., and Butler, M. J. R. (2012). The domain of organizational cognitive neuroscience: Theoretical and empirical challenges. Journal of Management, 38, 921-931.

Lee, L., Wong, P. K., Der Foo, M., and Leung, A. (2011). Entrepreneurial intentions: The influence of organizational and individual factors. Journal of Business Venturing, 26 (1), 124-136.

Li, Y., Chen, H., Liu, Y., and Peng, M. W. (2014). Managerial ties, organizational learning, and opportunity capture: A social capital perspective. Asia Pacific Journal of Management, 31 (1), 271-291.

Lim, D.S.K., Morse, E.A., Mitchell, R.K., and Seawright, K.K. (2010). Institutional environment and entrepreneurial cognitions: A comparative business systems perspective. Entrepreneurship Theory \& Practice, 4 (3), 491-501.

Lindquist, M.J., Sol, J., and Van Praag, M. (2012). Why do entrepreneurial parents have entrepreneurial children? (Tinbergen Institute Discussion Paper No. 12-062/3). Retrieved from http://ssrn.com/abstract=2101543 
Low, M. B., and MacMillan, I. C. (2007). Entrepreneurship: Past research and future challenges. In Entrepreneurship (pp. 131-154). Springer Berlin Heidelberg.

Louw, L., Van Eeden, S.M., Bosch, J.K., and Venter, D.J.L. (2003). Entrepreneurial traits of undergraduate students at selected South African tertiary Institutions. International Journal of Entrepreneurial Behaviour and Research, 9 (1), 5-26.

Lumpkin, G.T., and Dess, G.G. (1996). Clarifying the entrepreneurial orientation construct and linking it to performance. Academy of Management Review, 21 (1), 135-172.

Man, T. W. Y., and Lau, T. (2000). Entrepreneurial competencies of SME owner/managers in the Hong Kong services sector: A qualitative analysis. Journal of Enterprising Culture, 8 (3), 235-254.

Martin de Holan, P. (2014). It's all in your head: Why we need neuroentrepreneurship. Journal of Management Inquiry, 23 (1), 93-97.

Martínez-Campos, J. L., and Cantillo-Guerrero, E. F. (2013). Análisis de enfoques epistemológicos en tesis doctorales relacionadas con el marketing y el emprendimiento. Escenarios, 11 (2), 47-51.

Marvel, M. R. (2013). Human capital and search-based discovery: A study of high-tech entrepreneurship. Entrepreneurship theory and practice, 37 (2), 403-419.

Mayer, J. D., and Salovey, P. (1997). What is emotional intelligence?. In P. Salovey and D. Sluyter (eds.), Emotional development and emotional intelligence: Implications for educators (pp. 3-31). New York: Basic Books.

McClelland, D. C. (1961). The Achieving Society. Princeton. NJ: Van Nostrand.

McClelland, D. C. (1973). Testing for competence rather than intelligence. American Psychologist, 28 (1), 1-40.

McGrath, R. G., and Macmillan, I. (2000). The entrepreneurial mindset. Boston, MA.: Harvard Business School Press.

Momeni, N. (2009). The relation between managers' emotional intelligence and the organizational climate they create. Public Personnel Management, 38 (2), 35-48.

Moriano, J.A., Topa, G., Molero, F., Entenza, A.M., and Lévy-Mangin, J.P. (2012). Autoeficacia para el liderazgo emprendedor. Adaptación y validación de la escala CESE en España. Anales de Psicología, 28 (1), 171-179.

Mortan, R. A., Ripoll, P., Carvalho, C., and Bernal, M. C. (2014). Effects of emotional intelligence on entrepreneurial intention and self-efficacy. Revista de Psicología del Trabajo y de las Organizaciones, 30 (3), 97-104.

Naktiyok, A., Karabey, N. C., and Gulluce, A. C. (2010). Entrepreneurial self-efficacy and entrepreneurial intention: the Turkish case. International Entrepreneurship Management Journal, 6 (4), 419-435. 
Neghabi, S., Yousefi, M., and Rezvani, M. (2011). Emotional intelligence and entrepreneurial behavior: development of personal aspects of intentions model. International Conference on Information and Finance, 21, (pp. 27-31). Singapore: IACSIT Press.

Nezhad, H., Marjani, M., and Najafi, A. (2015). A study on relationship between emotional intelligence and entrepreneurship of trainees in vocational and technical organization. Management Science Letters, 5 (5), 501-506.

Nicolaou, N., and Shane, S. (2014). Biology, Neuroscience, and Entrepreneurship. Journal of Management Inquiry, 23 (1), 98-100.

North, D.C., 1990. Institutions, institutional change and economic performance. Cambridge, U.K.: Cambridge University Press.

0’Boyle, E.H., Jr., Humphrey, R.H., Pollack, J.M., Hawver, T.H., and Story, P. (2011). The relation between Emotional Intelligence and job performance: A metanalysis. Journal of Organizational Behavior, 32 (5), 788-818.

Pachulia, G., and Henderson, L. (2009). The relationship between emotional intelligence and entrepreneurial orientation; observed within owner-managers who lead small, high-tech firms in Sweden. Unpublished Master thesis, Jonkoping University.

Pérez, J.C., Petrides, K.V., and Furnham, A. (2005). Measuring trait emotional intelligence. In R. Schulze and Roberts, R.D. International Handbook of Emotional Intelligence. Cambridge: Hogrefe and Huber.

Petrides, K.V., and Furnham, A. (2001). Trait emotional intelligence: psychometric investigation with reference to established trait taxonomies. European Journal of Personality, 15 (6), 425-448.

Pihie, L., and Akmaliah, Z. (2009). Entrepreneurship as a career choice: An analysis of entrepreneurial self-efficacy and intention of university students. European Journal of Social Sciences, 9 (2), 338-349.

Pihie, A.Z.L., and Bagheri, A. (2010). Entrepreneurial attitude and entrepreneurial efficacy of technical secondary school students. Journal of Vocational Education \& Training, 62 (3), 351-366.

Pradham, R.K., and Nath, P. (2012). Perception of entrepreneurial orientation and emotional intelligence: A study on India's future techno-managers. Global Business Review, 13 (1), 89-108.

Rae, D. (2006). Entrepreneurial learning: A conceptual framework for technology-based enterprise. Technology Analysis \& Strategic Management, 18 (1), 39-56.

Rauch, A., and Frese, M. (2007). Let's put the person back into entrepreneurship research: A meta-analysis on the relationship between business owners' personality traits, business creation, and success. European Journal of Work and Organizational Psychology, 16 (4), 353-385. 
Rego, A., Sousa, F., Cunha, M.P., Correia, A., and Amaral, I.S. (2007). Leader self-reported emotional intelligence and perceived employee creativity: an exploratory study. Creativity and Innovation Management, 16 (3), 250-264.

Reynolds, P., Bosma, N., Autio, E, Hunt, S., De Bono, N., Servais, I., Lopez-Garcia, P. and Chin, N. (2005). Global Entrepreneurship Monitor: Data collection design and implementation 1998-2003. Small Business Economics, 24 (3), 205-231.

Rhee, K. S., and White, R. J. (2007). The Emotional Intelligence of Entrepreneurs. Journal of Small Business and Entrepreneurship, 20 (4), 409-426.

Rosete, D., and Ciarrochi, J. (2005). Emotional intelligence and its relationship to workplace performance outcomes of leadership effectiveness. Leadership \& Organization Development Journal, 26 (5), 388-399.

Rubin, R. S., Munz, D. C., and Bommer, W. H. (2005). Leading from within: The effects of emotion recognition and personality on transformational leadership behavior. Academy of Management Journal, 48 (5), 845-858.

Salvador, C. M. (2008). Impacto de la inteligencia emocional percibida en la autoeficacia emprendedora. Boletín de psicología, 92, 65-80.

Schnell, K., Bluschke, S., Konradt, B., and Walter, H. (2011). Functional relations of empathy and mentalizing: And fMRI study on the neural basis of cognitive empathy. NeuroImage, 54, 1743-1754.

Schumpeter, J. (1934). The Theory of Economic Development. Cambridge. Mass.: Harvard University Press.

Senior, C., Lee, N., and Butler, M. (2011). Organizational cognitive neuroscience. Organization Science, 22, 804-815.

Shane, S. (2003). A general theory of entrepreneurship. The individual-opportunity nexus. Cheltenham: Edward Elgar Publishing.

Shane, S., and Venkataraman, S. (2000). The promise of entrepreneurship as a field of research. Academy of Management Journal, 25 (1), 217-226.

Shepherd, D.A. (2011). Multilevel entrepreneurship research: opportunities for studying entrepreneurial decision making. Journal of Management, 37 (2), 412-420.

Siegling, A. B., Petrides, K. V., and Martskvishvili, K. (2015). An examination of a new psychometric method for optimizing multi-faceted assessment instruments in the context of trait emotional intelligence. European journal of personality, 29 (1), $42-54$.

Skudiene, V., Auruskeviciene, V., Reardon, J., and Stangej, 0. (2011). The effect of emotional intelligence on project outcomes. Transformation in Business \& Economics, 10, 1 (22), 125-137.

Sosa, M. (2008). Escala autoinformada de Inteligencia Emocional (EAIE). Tesis doctoral, Universidad Complutense de Madrid. 
Stephan, U., Uhlaner, L. M., and Stride, C. (2015). Institutions and social entrepreneurship: The role of institutional voids, institutional support, and institutional configurations. Journal of International Business Studies, 46, 308-331.

Stevenson, H. H., and Jarillo, J. C. (1990). A paradigm of entrepreneurship: entrepreneurial management. Strategic Management Journal, Special issue, 11, 17-27.

Stewart Jr. W. H., and Roth, P. L. (2001). Risk propensity differences between entrepreneurs and managers: a meta-analytic review. Journal of Applied Psychology, 86 (1), 145.

Stewart Jr. W. H., and Roth, P. L. (2007). A meta-analysis of achievement motivation differences between entrepreneurs and managers. Journal of Small Business Management, 45 (4), 401-421.

Stubbs Koman, E., and Wolff, S. B. (2008). Emotional intelligence competencies in the team and team leader: A multi-level examination of the impact of emotional intelligence on team performance. Journal of Management Development, 27 (1), 55-75.

Sunindijo, R.Y., Hadikusumo, B.H.W., and Ogunlana, S. (2007). Emotional intelligence and leadership styles in construction project management. Journal of Management in Engineering, 23 (4), 166-170.

Webb, C. A., Schwab, Z. J., Weber, M., DelDonno, S., Kipman, M., Weiner, M. R., and Killgore, W. D. S. (2013). Convergent and divergent validity of integrative versus mixed model measures of emotional intelligence. Intelligence, 41, 149-156.

Whitener, E. M., Brodt, S. E., Korsgaard, M. A., and Werner, J. M. (1998). Managers as initiators of trust: An exchange relationship framework for understanding managerial trustworthy behavior. Academy of Management Review, 23 (3), 513-530.

Winterton, J. (2002). Entrepreneurship: Towards a competence framework for developing SME managers. Paper presented at the United States Association for Small Business and Entrepreneurship Conference (pp. 12-20). Reno, Nevada: Conference Proceedings.

Wu, C., McMullen, J. S., Neubert, M. J., and Yi, X. (2008). The influence of leader regulatory focus on employee creativity. Journal of Business Venturing, 23 (5), 587-602.

Yang, H., and Dess, G.G. (2007). Where do entrepreneurial orientations come from, an investigation on their social origin. In Lumpkin, G.T., and Jerome A. Katz (ed.), Entrepreneurial Strategic Processes (pp. 223-247) (Advances in Entrepreneurship, Firm Emergence and Growth, 10). Elsevier Ltd.

Yitshaki, R. (2012). How do entrepreneurs' emotional intelligence and transformational leadership orientation impact new ventures' growth? Journal of Small Business \& Entrepreneurship, 25 (3), 357-374.

Zahra, S.A., Korri, J.S., and Yu, J. (2005). Cognition and international entrepreneurship: Implications for research on international opportunity recognition and exploitation. International Business Review, 14 (2), 129-146. 
Zakarevičius, P., and Župerka, A. (2010). Expression of emotional intelligence in development of students'entrepreneurship. Economics and Management, 15, 865-873.

Zampetakis, L.A., Beldekos, P., and Moustakis, V.S. (2009). Day-to-day entrepreneurship within organisations: The role of Trait Emotional Intelligence and perceived organisational support. European Management Journal, 27, 165-175.

Zampetakis, L.A., Kafetsios, K., Bouranta, N., Dewett, T., and Moustakis (2009). On the relationship between emotional intelligence and entrepreneurial attitudes and intentions. International Journal of Entrepreneurial Behaviour \& Research, 15 (6), 595-618.

Zhao, H., and Seibert, S.E. (2006). The Big Five personality dimensions and entrepreneurial status: A meta-analytical review. Journal of Applied Psychology, 91 (2), 259-271.

Zhao, H., Seibert, S. E., and Hills, G. E. (2005). The mediating role of self-efficacy in the development of entrepreneurial intentions. Journal of Applied Psychology, 90 (6), 1265.

Zhao, H., Seibert, S. E., and Lumpkin, G. T. (2010). The relationship of personality to entrepreneurial intentions and performance: A meta-analytic review. Journal of Management, 36 (2), 381-404.

Zhou, J., and George, J. M. (2003). Awakening employee creativity: The role of leader emotional intelligence. The Leadership Quarterly, 14 (4), 545-568.

Zuluaga, J.C. (2010). Emprendimiento e Historia Empresarial. Apuntes para un diálogo interdisciplinario en América Latina. Paper presented at the Congreso Latinoamericano de Historia Económica. Sesión II (pp. 1-32). Mexico D.F.: Conference Proceedings. 
